\title{
Perfil clínico-epidemiológico de traumatismo cranioencefálico do Hospital de Urgências e Traumas no município de Petrolina, estado de Pernambuco
}

\author{
José Carlos de Moura', Bruno Lázaro Ramos Rangel², Sheila Cristiane \\ Evangelista Creôncio ${ }^{3}$, José Ricardo Barros Pernambuco ${ }^{4}$
}

Hospital de Urgências e Traumas Dr. Washington Antônio de F. Barros.

\section{RESUMO}

Objetivo: No Brasil, dentre o conjunto de lesões decorrentes das causas externas, o traumatismo cranioencefálico (TCE) destaca-se em termos de magnitude, tanto em mortos quanto em feridos. Este estudo visa avaliar epidemiologicamente os casos de TCEs ocorridos no Vale do São Francisco, analisando-os quanto a idade, sexo, procedência, etiologia do trauma, conduta e exames complementares. Método: Foram estudados, de forma descritiva e analítica, com abordagem quantitativa, os prontuários dos pacientes vítimas de TCE admitidos no Hospital de Urgências e Traumas, entre os meses de dezembro de 2008 a junho de 2009. No total, foram avaliados 101 prontuários. Conclusão: A etiologia do TCE mais frequente foram os acidentes motociclísticos, seguidos por queda. O sexo masculino é o mais acometido e a faixa etária mais acometida foi a dos 21 aos 40 anos. Com relação à classificação de gravidade do TCEs, 53,47\% foram classificados como leves, $25,73 \%$, como moderados e $20,80 \%$, como graves; evidencia-se a prevalência do TCE leve, porém com percentual significativo de casos graves.

\section{PALAVRAS-CHAVE}

Traumatismos encefálicos, acidentes de trânsito, traumatismos do sistema nervoso.

\section{ABSTRACT}

Clinical and epidemiological profile of head trauma at the Urgências e Traumas Hospital in the city of Petrolina, Pernambuco

Objective: In Brazil, among the set of injuries resulting from external causes, the head trauma (HT) stands out in terms of magnitude, both in dead and wounded. This study aims to evaluate epidemiologically the cases of traumatic brain injury occurred in the São Francisco Valley analyzing them in age, sex, origin, trauma etiology, conduct and additional tests. Method: The medical records of patients victims of head injury admitted to the Urgências e Traumas Hospital, between December 2008 and June 2009 were studied in a descriptive and analytical with a quantitative approach. A total of 101 medical records were evaluated. Conclusion: The most common etiology of HT were motorcycle accidents, followed by fall. Male is the most affected gender and most affected age group was 21 to 40 years. Regarding the classification of severity of $H T, 53.47 \%$ were classified as mild, $25.73 \%$ were classified as moderate and $20.80 \%$ classified as severe, indicating the prevalence of mild HT, but with a significant percentage of severe cases.

\section{KEYWORDS}

Brain injuries, accidents, traffic, trauma, nervous system.

1. Universidade Federal do Vale do São Francisco (Univasf), Serviço de Neurologia e Neurocirurgia do Hospital Neurocardio e do Hospital de Urgências e Traumas, Petrolina, PE, Brasil.

2. Univasf, Petrolina, PE, Brasil.

3. Hospital de Urgências e Traumas, Petrolina, PE, Brasil.

4. Univasf, Hospital de Urgências e Traumas, Petrolina, PE, Brasil. 


\section{Introdução}

No Brasil, no conjunto de lesões decorrentes das causas externas, o traumatismo cranioencefálico (TCE) destaca-se em termos de magnitude, tanto em mortos quanto em feridos, sendo uma das lesões mais frequentes. ${ }^{9} \mathrm{O}$ TCE constitui-se como um dos principais problemas de saúde pública mundial, apresentando elevada e crescente incidência no mundo moderno e representando uma importante causa de morbimortalidade em adolescentes e adultos jovens, com consequentes perdas da capacidade produtiva e prejuízos financeiros para a sociedade. ${ }^{4,5}$ Tem-se observado, ainda, um aumento da mortalidade resultante de TCE, particularmente nos países em vias de desenvolvimento. ${ }^{22}$

Nos últimos 10 anos, mais de 1 milhão de pessoas ficaram inválidas devido a traumas mecânicos no Brasil, sendo os acidentes de trânsito os principais responsáveis por essas taxas. A depender do hospital estudado, o internamento por trauma mecânico pode atingir valores acima de 40\%. ${ }^{6}$ Dados de 2004 da Organização Mundial de Saúde (OMS) mostram que os acidentes de trânsito são responsáveis por aproximadamente 1,2 milhão de mortes por ano e causam lesões em cerca de 50 milhões. ${ }^{16}$

Além do aumento do número de veículos em circulação, a desorganização, a deficiência geral da fiscalização, as péssimas condições de muitos veículos, o comportamento dos usuários e a impunidade dos infratores fizeram com que nas últimas décadas o Brasil se colocasse entre os campeões mundiais de acidentes de trânsito. ${ }^{15}$

A incidência geral de TCE varia de acordo com o sexo, sendo mais frequente nos homens do que nas mulheres, o que pode refletir diferenças nas situações de risco. Um estudo de revisão de Kraus e Mcarthur ${ }^{10}$, que compara homens com mulheres, refere uma proporção de cerca de 2 a 2.8:1. A proporção relativa à mortalidade é ainda mais discrepante, aproximadamente de 3.5:1, indicando maior gravidade de lesão nos homens (dados dos Estados Unidos). ${ }^{22}$

A mortalidade por TCE está relacionada com a idade, verificando-se um número maior de mortos entre sujeitos mais velhos. Entre as crianças, os TCEs são referidos como a principal causa de morte em resultado de acidentes traumáticos. Porém, as crianças têm uma taxa de mortalidade menor do que os adultos. ${ }^{22}$

Entre as principais causas de TCE, podem-se citar acidentes automobilísticos, atropelamentos, acidentes ciclísticos e motociclísticos, agressões físicas, quedas, lesões por arma de fogo, entre outras menos frequentes. ${ }^{12} \mathrm{O}$ Brasil, nos últimos anos, vem registrando um crescimento exacerbado na frota de motocicletas, $o$ que vem implicando aumento crescente no número de acidentes envolvendo esse tipo de veículo.
Entende-se por TCE qualquer agressão de ordem traumática que acarrete lesão anatômica ou comprometimento funcional do couro cabeludo, crânio, meninges, encéfalo ou seus vasos. ${ }^{11}$

Estudos demonstram que no Brasil o TCE leve é responsável por cerca de $80 \%$ dos casos $^{12,22}$ em adultos e $63,8 \%$ em adolescentes. ${ }^{13}$ Segundo Melo et al. ${ }^{12}$, no Brasil, mais de 1 milhão de pessoas ficaram com sequelas neurológicas irreversíveis devido à ocorrência de TCE, nos últimos dez anos.

Sendo assim, é de fundamental importância o conhecimento das características locais, para que sejam, então, adotadas medidas de prevenção mais efetivas baseadas nessa realidade, já que as causas primárias do TCE variarão de acordo com a população envolvida. $\mathrm{O}$ presente estudo pretende ajudar no conhecimento das características epidemiológicas de TCE nessa região.

\section{Metodologia}

Foram estudados os prontuários dos pacientes admitidos no serviço do Hospital de Urgências e Traumas Dr. Washington Antônio de F. Barros (HUT), acometidos por TCE, na região do Vale do São Francisco, entre o período de dezembro de 2008 e junho de 2009 . O protocolo de pesquisa foi aprovado pelo Comitê Institucional de Ética em Pesquisa da Universidade Federal do Vale do São Francisco, sob parecer no ${ }^{\circ}$ 013/2010.

Foram analisadas as seguintes variáveis conforme protocolo estabelecido: sexo, idade, profissão, raça, procedência, data de admissão, etiologia do trauma, área craniana lesada, associação com bebida alcoólica, sinais e sintomas à admissão, escore da ECG no momento da admissão e classificação de gravidade do TCE, achados tomográficos, tipo de tratamento instituído, tempo de internamento, alta hospitalar e óbito.

Após a coleta das informações, elas foram submetidas a uma análise estatística descritiva, em que os dados foram transferidos para planilhas do Microsoft Excel ${ }^{\circledR} \mathrm{e}$ Microsoft Word ${ }^{\circledR}$, para construção dos gráficos e tabelas, contendo números absolutos e percentuais seguidos de análise e discussão dos resultados.

Durante a realização desta pesquisa, não foram declarados conflitos de interesse associados à realização deste estudo.

\section{Resultados}

Foi estudada uma amostra de 101 prontuários de pacientes vítimas de TCE. O sexo masculino foi o mais 
acometido, com 87 casos (86,14\%), e 14 casos (13,86\%) do sexo feminino (Figura 1). Houve maior incidência na faixa etária dos 21 aos 40 anos, totalizando 51,49\% (Tabela 1). Não houve informações nos prontuários acerca da profissão dos pacientes.

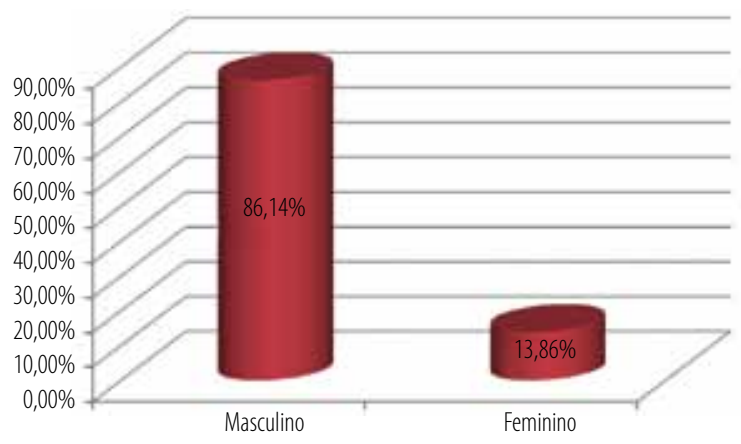

Figura 1 - Incidência por sexo.

Tabela 1 - Incidência por idade (em anos)

\begin{tabular}{rr}
$0-20$ & $21,78 \%$ \\
$21-40$ & $51,49 \%$ \\
$41-60$ & $15,84 \%$ \\
$61-80$ & $8,91 \%$ \\
80 ou mais & $1,98 \%$ \\
\hline
\end{tabular}

As principais causas de TCE foram: 45 casos $(44,55 \%)$ por acidente motociclístico, 23 casos $(22,78 \%)$ por queda, 12 casos $(11,88 \%)$ por atropelamento, $10 \mathrm{ca}-$ sos $(9,9 \%)$ por acidente automobilístico, 6 casos $(5,94 \%)$ por agressão física e outros casos não especificados somaram cinco casos $(4,95 \%)$. (Figura 2) Quanto à gravidade do TCE, 54 (53,47\%) foram leves, 26 (25,73\%), moderados e 21 (20,8\%), graves. (Figura 3 )

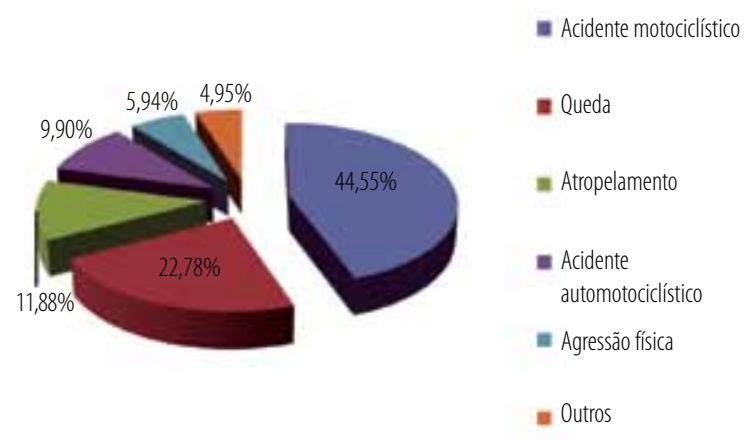

Figura 2 - Etiologia do trauma.

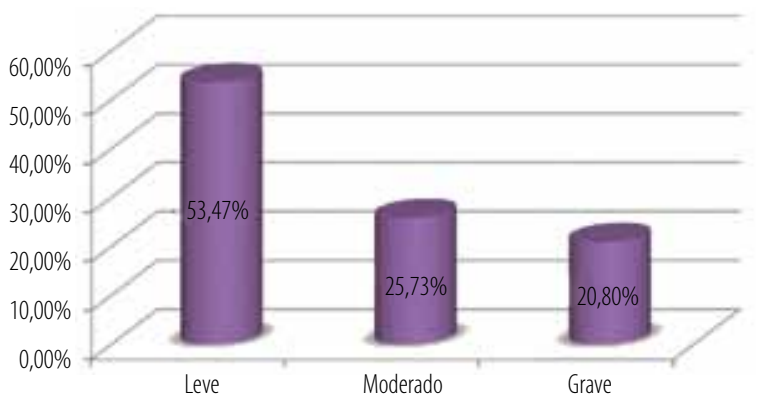

Figura 3 - Gravidade do TCE.

No universo dos acidentes motociclísticos, houve 22 $(51,16 \%)$ casos de TCE leve, 12 (27,91\%), moderado e 9 (20,93\%), grave. Dentre os acidentes por queda, houve $14(66,67 \%)$ casos de TCE leve, $4(19,04 \%)$ moderado e $3(14,29 \%)$ grave. Em se tratando de acidente automobilístico, houve $6(60 \%)$ casos de TCE leve, $1(10 \%)$ moderado e 3 (30\%) grave. Quanto aos atropelamentos, houve $5(38,46 \%)$ casos de TCE leve, $4(30,77 \%)$ moderado e $4(30,77 \%)$ grave. Com relação às agressões físicas, houve $2(33,33 \%)$ casos de TCE leve, 3 (50\%) moderado e $1(16,67 \%)$ grave.

Quanto à procedência dos pacientes, os municípios foram assim distribuídos: Petrolina com 69 (68,32\%) casos e cidades vizinhas somando $32(31,68 \%)$ casos (Tabela 2).

\begin{tabular}{lcc}
\hline \multicolumn{3}{c}{ Tabela 2 - Cidade de origem e números de casos } \\
\hline Petrolina & 69 & $68,32 \%$ \\
Outras cidades & 32 & $31,68 \%$ \\
TOTAL & 101 & $100 \%$ \\
\hline
\end{tabular}

O exame neurológico apresentou-se alterado na maioria dos casos. Os principais sinais clínicos apresentados foram: alteração do nível de consciência com 38 casos $(37,62 \%)$, cefaleia com 17 casos $(16,83 \%)$, vômito com 16 casos $(15,84 \%)$, otorragia com 9 casos $(8,91 \%)$ e coma com 6 casos $(5,94 \%)$ (Tabela 3$)$.

\begin{tabular}{lcc}
\hline \multicolumn{3}{c}{ Tabela 3 - Sinais clínicos prevalentes na } \\
admissão e número de casos \\
\hline Diminuição do nível de consciência & $\mathbf{3 8}$ & $\mathbf{3 7 , 6 2 \%}$ \\
Cefaleia & 17 & $16,83 \%$ \\
Vômito & 16 & $15,84 \%$ \\
Otorragia & 9 & $8,91 \%$ \\
Coma & 6 & $5,94 \%$ \\
\hline
\end{tabular}


Na nossa casuística, em 50 prontuários $(49,5 \%)$ havia a referência documentada sobre uso de bebida alcoólica (Tabela 4).

\begin{tabular}{lcc}
\hline \multicolumn{3}{c}{ Tabela 4 - Uso de bebida alcoólica e } \\
número de casos documentados \\
\hline \multirow{2}{*}{ Não } & 50 & $49,5 \%$ \\
Sim & 51 & $50,5 \%$ \\
Total & 101 & $100 \%$ \\
\hline
\end{tabular}

Dentre os exames complementares, 11 pacientes $(10,89 \%)$ realizaram apenas radiografia de crânio, 38 $(37,62 \%)$ realizaram apenas tomografia computadorizada (TC) de crânio, 49 (48,51\%) realizaram ambos os exames, enquanto 3 (2,98\%) não realizaram nenhum dos dois tipos de exames (Figura 4). O domingo foi o dia da semana em que ocorreu o maior número de atendimentos por TCE, com 25 casos (24,75\%), seguido pela segunda-feira, com 18 casos $(17,82 \%)$; os outros dias somam $58(57,43 \%)$ casos (Tabela 5).
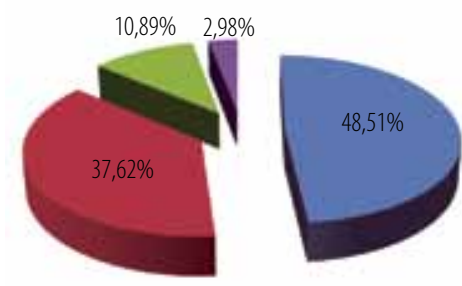

= TC e Rx de crânio

$=\mathrm{TC}$

= Rx de crânio

= Não realizou exame

Figura 4 - Exames complementares realizados.

Tabela 5 - Dias do atendimento e número de casos

\begin{tabular}{lcc}
\hline Domingo & 25 & $24,75 \%$ \\
Segunda-feira & 18 & $17,82 \%$ \\
Outros dias & 58 & $57,43 \%$ \\
Total & 101 & $100 \%$ \\
\hline
\end{tabular}

A área craniana lesada mais frequente em nossa pesquisa foi a frontal, com $24,75 \%$, seguida pela temporal e temporoparietal, com $11,88 \%$ cada uma, parietal, com $8,91 \%$, occipital e parietofrontal, com $5,94 \%$ cada uma, frontotemporal, com 3,96\%, e temporo-occipital e fratura de base do crânio, com 1,98\% cada uma (Tabela 6). Em 22,78\% dos prontuários não havia essa informação.

Com relação aos achados tomográficos, obteve-se maior incidência de hematoma extradural (HED), com $19,82 \%$, seguido por contusão cerebral, com $17,82 \%$, hemorragia subaracnoidea (HSA), com 9,9\%, hematoma subdural (HSD), com 5,94\%, e lesão axonal difusa (LAD), com $0,99 \%$ dos casos. Dos pacientes que foram submetidos à TC, 61,39\% tiveram alguma alteração, $17,82 \%$ não apresentaram nenhuma alteração e em 20,79\% das fichas não havia o laudo (Figura 5).

\begin{tabular}{lcc}
\hline \multicolumn{3}{c}{ Tabela 6 - Área craniana lesada e número de casos } \\
\hline Frontal & 25 & $24,75 \%$ \\
Temporal & 12 & $11,88 \%$ \\
Temporoparietal & 12 & $11,88 \%$ \\
Parietal & 9 & $8,91 \%$ \\
Occipital & 6 & $5,94 \%$ \\
Parietofrontal & 6 & $5,94 \%$ \\
Frontotemporal & 4 & $3,96 \%$ \\
Temporo-occipital & 2 & $1,98 \%$ \\
Fratura de base de crânio & 2 & $1,98 \%$ \\
Sem informação & 23 & $22,78 \%$ \\
Total & 101 & $100 \%$ \\
\hline
\end{tabular}

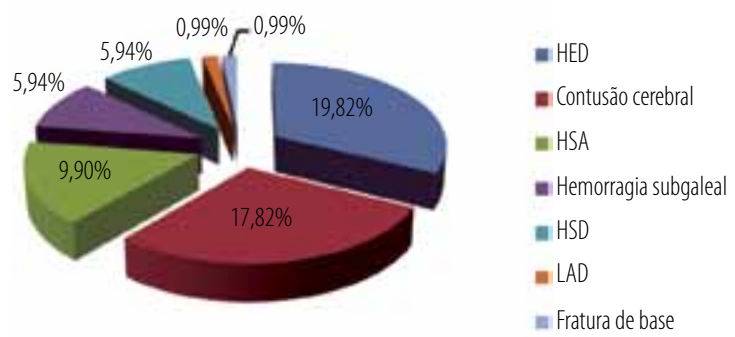

Figura 5 - Achados tomográficos.

Em relação ao tipo de tratamento instituído, 71,29\% dos casos receberam tratamento clínico, enquanto $28,71 \%$ receberam tratamento cirúrgico. A média de tempo de internamento de cada paciente foi de 5,99 dias. Quanto ao desfecho, 88,12\% dos casos receberam alta, enquanto 7,92\% evoluíram para o óbito (Figura 6).

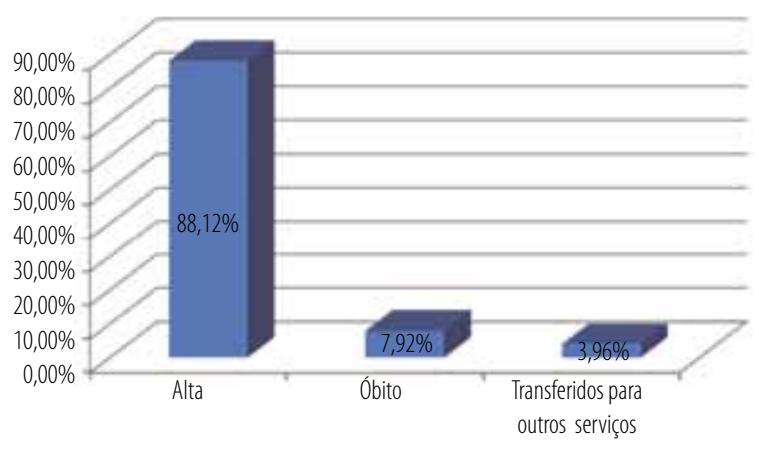

Figura 6 - Evolução. 


\section{Discussão}

Conhecer o perfil dos pacientes atendidos em cada serviço é essencial, pois o esclarecimento dos dados epidemiológicos, sinais e sintomas clínicos, tipos e resultados de exames de imagem realizados, tempo de permanência hospitalar, procedimentos cirúrgicos e evolução clínica antes e após a alta é um passo essencial para o planejamento de ações preventivas e para a melhoria do atendimento.

Dessa maneira, para que fosse conhecido o perfil dos pacientes que sofreram TCE do HUT, foram analisados os prontuários dos meses de dezembro do ano de 2008 a junho do ano de 2009. Soares e Barros ${ }^{23}$ sugerem que a implantação e a manutenção de medidas de segurança no trânsito sejam efetivas para diminuir a gravidade dos acidentes, tendo em vista a redução de óbitos por TCE; para isso, é necessário conhecer o perfil dessas vítimas, a fim de intervir de forma mais direcionada.

No presente estudo, houve predominância do sexo masculino, correspondendo a $86,14 \%$ das vítimas, semelhante a outros estudos publicados. ${ }^{3,7,8,13,17,18,20,21} \mathrm{Em}$

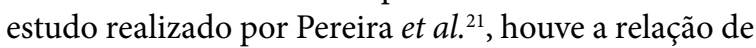
2,7:1 em relação à incidência do sexo masculino sobre o feminino. Neste trabalho, a relação encontrada foi de 6,21:1, o que denota a peculiaridade de nossa amostra.

A exemplo de vários outros estudos, ${ }^{1,3,8,13,17,18,20,21} \mathrm{em}$ nossa amostra prevaleceu a faixa etária dos 21 aos 40 anos, com 51,49\%. Com relação à procedência, a maior parte dos casos era advinda do município de Petrolina, com $68,32 \%$ do casos.

Nesta pesquisa, a etiologia do trauma que prevaleceu foi a de acidentes motociclísticos, com 44,55\% do total da amostra, seguidos por queda, com 22,78\%; 11,88\% foram vítimas de atropelamento, $9,9 \%$ sofreram acidente automobilístico, 5,94\% sofreram agressão física e outros casos somaram 4,95\% dos casos. Essa maior prevalência de TCE por acidente motociclístico pode ser explicada pelo grande aumento dos usuários desse tipo de transporte, além de esse fato ser também corroborado por outros estudos. $3 ., 8,23$

Em 49,5\% dos casos, houve registro do uso ou não de bebidas alcoólicas antes do acidente. Alguns outros estudos também demonstram que a bebida alcoólica está associada à ocorrência dos acidentes. ${ }^{3}$

Dos pacientes atendidos no período do estudo, $71,29 \%$ receberam tratamento clínico, enquanto os que receberam tratamento cirúrgico somaram $28,71 \%$. A exemplo da nossa pesquisa, Melo et al. ${ }^{13}$ encontraram maior prevalência do tratamento clínico. Em um estudo realizado por Pereira et al. ${ }^{18}$ houve predominância do tratamento cirúrgico, pois todos os pacientes sofreram hematoma extradural frontal traumático confirmado por TC. A média de internamento dos pacientes da nossa casuística foi de 5,99 dias, média maior do que a encontrada por Franciozi et al. ${ }^{7}$ que foi de 4,1 dias.

$\mathrm{O}$ uso de métodos diagnósticos adequados é essencial para minimizar custos e melhorar o resultado do tratamento a ser instituído ao paciente traumatizado, sendo fundamental para o estabelecimento de medidas terapêuticas clínicas e/ou cirúrgicas. ${ }^{2}$ Mesmo quando qualquer método diagnóstico for instituído, é essencial o monitoramento clínico contínuo do paciente. ${ }^{14}$

Embora existam lesões que não sejam detectadas pela TC, ela no momento é a maneira inicial mais rápida para a detecção de lesões com necessidade de intervenção cirúrgica. ${ }^{14}$ Houve em nossa casuística maior frequência de lesões da área craniana frontal e o achado tomográfico mais incidente foi o HED. Dos pacientes que foram submetidos à TC, 61,39\% tiveram alguma alteração, 17,82\% não apresentaram nenhuma alteração e em 20,79\% não havia a descrição. Pereira et al., ${ }^{21} \mathrm{em}$ seu estudo, constataram que apenas $31 \%$ dos casos apresentaram alterações tomográficas.

Com relação à classificação de gravidade do TCE, foi utilizada a Escala de Coma de Glasgow (ECG), na qual $53,47 \%$ foram classificados como leves, $25,73 \%$, como moderados e 20,80\%, como graves. A exemplo de outras pesquisas, prevaleceu o TCE leve. ${ }^{3,13,19,21,23}$ Diferente de um estudo realizado por Braga et al., ${ }^{3}$ obteve-se neste trabalho um maior número de atendimentos entre domingo e quarta-feira, sendo domingo o dia de maior atendimento por TCE, com $24,75 \%$, fato curioso em nossa pesquisa.

Com relação à evolução do trauma, $88,12 \%$ pacientes receberam alta hospitalar; $7,92 \%$ dos casos evoluíram para óbito e os que foram transferidos para outros serviços hospitalares somaram 3,96\% dos casos. Esses dados obtidos são semelhantes aos encontrados por outros autores. ${ }^{3,8,21}$

\section{Conclusão}

A etiologia do TCE mais frequente foi a de acidentes motociclísticos, seguidos por queda. O sexo masculino é o mais acometido e a faixa etária mais acometida foi a dos 21 aos 40 anos.

Com relação à classificação de gravidade do TCE, $53,47 \%$ foram classificados como leves, $25,73 \%$, como moderados e $20,80 \%$, como graves, evidenciando a prevalência do TCE leve, porém com percentual significativo de casos graves. 


\section{Agradecimentos}

Os autores agradecem aos funcionários do Hospital de Urgências e Traumas pela contribuição para a realização desta pesquisa.

\section{Conflitos de interesse}

Não foram declarados conflitos de interesse associados à publicação deste artigo.

\section{Referências}

1. Alho EJL, Paiva WS, Amorim RLO, Figueiredo EG, Andrade AF, Teixeira MJ. Hemorragia subaracnóidea traumática: aspectos clínicos, radiológicos e complicações. J Bras Neurocirurg. 2008;19(3):31-6.

2. Andrade AF, Paiva WS, Amorim RLO, Figueiredo EG, Neto ER, Teixeira MJ. Mecanismos de lesão cerebral no traumatismo cranioencefálico. Rev Assoc Med Bras. 2009;55(1):75-81.

3. Braga FM, Netto AA, Santos ER, Braga PB. Avaliação de 76 casos de traumatismo cranioencefálico por queda da própria altura atendidos na emergência de um hospital geral. ACM Arq Catarin Med. 2008;37(4):35-9.

4. Fakhry SM, Trask AL, Waller MA, Watts DD; IRTC Neurotrauma Task Force. Management of brain-injured patients by an evidence-based medicine protocol improves outcomes and decreases hospital charges. J Trauma. 2004;56(3):492-9.

5. Figg RE, Burry TS, Vander Kolk WE. Clinical efficacy of serial computed tomographic scanning in severe closed head injury patients. J Trauma. 2003;55(6):1061-4.

6. Fonseca A. Trânsito deixou um milhão de inválidos. Jornal da Tarde, caderno 01, p. 03, em 17 de julho de 2002.

7. Franciozi CES, Tamaoki MJS, Araújo EFA, Dobashi ET, Utumi CE, Pinto JA, et al. Trauma na infância e adolescência: epidemiologia, tratamento e aspectos econômicos em um hospital público. Acta Ortop Bras. 2008;16(5):261-5.

8. Gawryszewski VP, Coelho HM, Scarpelini S, Zan R, Jorge $\mathrm{MH}$, Rodrigues EM. Perfil dos atendimentos a acidentes de transporte terrestre por serviços de emergência em São Paulo, 2005. Rev Saude Publica. 2009;43(2):275-82.

9. Koizumi MS, Lebrão ML, Mello-Jorge MH, Primerano V. Morbimortalidade por traumatismo cranioencefálico no município de São Paulo, 1997. Arq Neuropsiquiatr. 2000;58(1):81-9.
10. Kraus JF, Mcarthur DL. Epidemiology of brain injury. In: Evans RW, editor. Neurology and trauma. Philadelphia: WB Saunders Company; 1996. p. 3-17.

11. Macedo KC. Características clínicas e epidemiológicas de crianças e adolescentes com traumatismo cranioencefálico leve e análise de fatores associados à fratura de crânio e lesão intracraniana [tese]. Belo Horizonte: Universidade Federal de Minas Gerais, Faculdade de Medicina; 2006.

12. Melo JR, Silva RA, Moreira ED Jr. Características dos pacientes com trauma cranioencefálico na cidade do Salvador, Bahia, Brasil. Arq Neuropsiquiatr. 2004;62(3A):711-5.

13. Melo JRT, Lemos-Júnior LP, Matos LT. Principais causas de trauma craniencefálico na cidade de Salvador, Bahia, Brasil. Arq Bras Neurocir. 2005;24(3):94-8.

14. Morais DF, SpottAR, Tognola WA. Contribuição da ressonância magnética encefálica no traumatismo craniencefálico fechado. Arq Bras Neurocir. 2006;25(3):125-31.

15. Oliveira NLB, Sousa RMC. Diagnóstico de lesões e qualidade de vida de motociclistas, vítimas de acidentes de trânsito. Rev Latino-am Enfermagem. 2003;11(6):749-56.

16. Organización Mundial de la Salud. Informe mundial sobre prevención de los traumatismos causados por el tránsito. Oficina regional de la Organización Mundial de la Salud. Publicación científica y técnica n.599. Disponível em: www.who.int/violence_injury_prevention/publications/ road_traffic/world_report/es/index.html. Acesso em: 16 ago. 2009.

17. Pereira CU, Leão JDBC, Silva AD, Bispo DJS, Santos EAS. Hematoma subdural inter-hemisférico traumático agudo. Arq Bras Neurocir. 2004;23(4):157-62.

18. Pereira CU, Leão JDBC, Ribas A, Santos EAS, Monteiro JTS, Duarte GC. Frontal epidural haematoma. Analysis of 30 cases. J Bras Neurocirurg. 2004;15(1):18-21.

19. Pereira CU, Santos EAS, Cavalcante S, Serra MV, Pascotto $D$, Fontoura EAF, et al. Hematoma extradural intracraniano. J Bras Neurocirurg. 2005;16(1):25-34.

20. Pereira CU, Barreto AS, Moreira LCM. Traumatismos craniencefálicos leves. Estudo comparativo entre observação clínica e realização de exames complementares. Arq Bras Neurocir. 2005;24(2):58-66.

21. Pereira CU, Duarte GC, Santos EAS. Avaliação epidemiológica do traumatismo craniencefálico no interior do estado de Sergipe. Arq Bras Neurocir. 2006;25(1):8-16.

22. Santos ME, De Sousa L, Castro-Caldas A. Epidemiologia dos traumatismos cranioencefálicos em portugal. Acta Med Port. 200;16(2):71-6.

23. Soares DFPP, Barros MBA. Gravidade dos acidentes de trânsito ocorridos em Maringá, PR. Ciência, Cuidado e Saúde Maringá. 2006;5(Supl):77-84.

Endereço para correspondência

José Carlos de Moura

Rua Joaquim Nabuco, 798, Centro

56304-040 - Petrolina, PE, Brasil

E-mail: jcdemoura@uol.com.br 